\title{
Quantum phase properties of photon added and subtracted displaced Fock states
}

\author{
Priya Malpani, ${ }^{1}$ Kishore Thapliyal, ${ }^{2,3}$ Nasir Alam, ${ }^{3}$ Anirban Pathak,${ }^{3}$ V. Narayanan, ${ }^{1}$ Subhashish Banerjee ${ }^{1}$ \\ ${ }^{1}$ Indian Institute of Technology Jodhpur, Jodhpur 342037, India \\ ${ }^{2}$ RCPTM, Joint Laboratory of Optics of Palacky University and Institute of \\ Physics of Academy of Science of the Czech Republic, Faculty of Science, \\ Palacky University, 17. listopadu 12, 77146 Olomouc, Czech Republic \\ ${ }^{3}$ Jaypee Institute of Information Technology, A-10, Sector-62, Noida UP-201309, India
}

\begin{abstract}
Quantum phase properties of photon added and subtracted displaced Fock states (and a set of quantum states which can be obtained as the limiting cases of these states) are investigated from a number of perspectives, and it is shown that the quantum phase properties are dependent on the quantum state engineering operations performed. Specifically, the analytic expressions for quantum phase distributions and angular $Q$ distribution as well as measures of quantum phase fluctuation and phase dispersion are obtained. The uniform phase distribution of the initial Fock states is observed to be transformed by the unitary operation (i.e., displacement operator) into non-Gaussian shape, except for the initial vacuum state. It is observed that the phase distribution is symmetric with respect to the phase of the displacement parameter and becomes progressively narrower as its amplitude increases. The non-unitary (photon addition/subtraction) operations make it even narrower in contrast to the Fock parameter, which leads to broadness. The photon subtraction is observed to be a more powerful quantum state engineering tool in comparison to the photon addition. Further, one of the quantum phase fluctuation parameters is found to reveal the existence of antibunching in both the engineered quantum states under consideration. Finally, the relevance of the engineered quantum states in the quantum phase estimation is also discussed, and photon added displaced Fock state is shown to be preferable for the task.
\end{abstract}

\section{INTRODUCTION}

Generation of the desired nonclassical states (i.e., quantum states without any classical counterpart), characterized by negative Glauber-Sudarshan $P$ function [1,2], from a given initial state can be performed with the help of both unitary dynamics and non-unitary operations. The unitary time evolution under the control of a Hamiltonian may result in the nonclassical states [3-7], but experimentally this is often limited to a small set of implementable Hamiltonians. Another approach, which includes non-unitary measurement or photon addition/subtraction operations, is known to be useful in some cases for generation of such states. A general recipe including both unitary and non-unitary operations, which enables us to generate a large set of nonclassical states in experiments, is labeled as quantum state engineering [8-14].

Particularly interesting examples of such engineered nonclassical states are Fock state, photon added/subtracted coherent state [15], displaced Fock state (DFS) [16-19], photon added DFS (PADFS), and photon subtracted DFS (PSDFS) [20]. Here, we address the relevance of such engineered quantum states in implementation of different tasks exploiting their phase properties. Our specific interest is to study the phase properties of PADFS and PSDFS, also known as photon added and subtracted generalized coherent states [20], as a large set of engineered quantum states (along with the conventional coherent state) can be obtained in the limiting cases of PADFS and PSDFS. In the recent past, the nonclassical properties of this set of engineered quantum states, many of which have been experimentally generated [19, 21-24], were focus of various studies (see [20] and references therein). However, in the present work, we do not want to focus on the specific nonclassical properties of these states. Rather, we wish to investigate the phase properties of these states for the reasons explained below.

The impossibility of writing a Hermitian operator for quantum phase is a longstanding problem (see [25-27] for review). Early efforts of Dirac [28] to introduce a Hermitian quantum phase operator were not successful, but led to many interesting proposals [29-31]. Specifically, Susskind-Glogower [29], Pegg-Barnett [30, 32, 33], and BarnettPegg [31] formalisms played very important role in the studies of phase properties and the phase fluctuation [34]. Thereafter, phase properties of various quantum states have been reported using these formalisms [26, 35-42]. Other approaches have also been used for the study of the phase properties. For example, quantum phase distribution is defined using phase states [43], while Wigner [44] and $Q[45,46]$ phase distributions are obtained by integrating over radial parameter of the corresponding quasidistribution function. In experiments, the phase measurement is obtained by averaging the field amplitudes of the $Q$ function [47, 48]; Pegg-Barnett and Wigner phase distributions are also reported with the help of reconstructed density matrix [49]. Further, quantum phase distribution under the effect of the environment was also studied in the past leading to phase diffusion [50-53]. A measure of phase fluctuation named phase dispersion using quantum phase distribution has also been proposed in the past $[25,50]$. Recently, quantum phase fluctuation [54] and Pancharatnam phase [55] have been studied for DFS. The quantum phase fluctuation in 
parametric down-conversion [56] and its revival [57] are also reported. Experiments on phase super-resolution without using entanglement [58] and role of photon subtraction in concentration of phase information [59] are also performed. Optimal phase estimation [60] using different quantum states [61] (including NOON and other entangled states and unentangled single-photon states) has long been the focus of quantum metrology [62, 63]. More recently, some efforts have been made to describe quasidistribution of phase as filtering in the interval of phase difference [64]. In brief, quantum phase properties are of intense interest of the community since long (see [25, 65] and references therein), and the interest in it has been further intensified in the recent past as many new applications of quantum phase distribution and quantum phase fluctuation have been realized.

To stress on the recently reported applications of quantum phase distribution and quantum phase fluctuation, we note that these have applications in quantum random number generation [66, 67], cryptanalysis of squeezed state based continuous variable quantum cryptography [68], generation of solitons in a Bose-Einstein condensate [69], storage and retrieval of information from Rydberg atom [70], in phase encoding quantum cryptography [71], phase imaging of cells and tissues for biomedical application [72]; as well as have importance in determining the value of transition temperature for superconductors [73]. Keeping these applications and the general nature of engineered quantum states PADFS and PSDFS in mind, we study phase distribution, $Q$ phase, phase fluctuation measures, phase dispersion, and quantum phase estimation using the concerned states and the states obtained in the limiting cases.

The rest of the paper is organized as follows. In Section II, we briefly introduce various parameters (e.g., quantum phase distribution, angular $Q$ function, quantum phase fluctuation measures, and uncertainty in quantum phase estimation) which are used in this paper to study the quantum phase properties. In Section III, we describe the PADFS, PSDFS, and a set of quantum states that can be obtained in the limiting cases. In Section IV, we investigate the phase properties of PADFS and PSDFS from a number of perspectives. Finally, the paper is concluded in Section V.

\section{QUANTUM PHASE DISTRIBUTION AND OTHER PHASE PROPERTIES}

Quantum phase operator $\hat{\phi}$ was introduced by Dirac based on his assumption that the annihilation operator $\hat{a}$ can be factored out into a Hermitian function $f(\hat{N})$ of the number operator $\hat{N}=\hat{a}^{\dagger} \hat{a}$ and a unitary operator $\hat{U}[28]$ as

$$
\hat{a}=\hat{U} f(\hat{N})
$$

where

$$
\hat{U}=e^{\iota \hat{\phi}}
$$

However, there was a problem with the Dirac formalism of phase operator as it failed to provide a meaning to the corresponding uncertainty relation. Specifically, in the Dirac formalism, the creation $\left(\hat{a}^{\dagger}\right)$ and annihilation $(\hat{a})$ operators

satisfy the bosonic commutation relation, $\left[\hat{a}, \hat{a}^{\dagger}\right]=1$, iff $[\hat{N}, \hat{\phi}]=\iota$, which leads to the number phase uncertainty relation $\Delta N \Delta \phi \geq 1$. Therefore, in order to satisfy the bosonic commutation relation under Dirac formalism, the phase uncertainty should be greater than $2 \pi$ for $\Delta N$ i $\frac{1}{2 \pi}$ which lacks a physical description. Subsequently, Louisell [74] proposed some periodic phase based method, which was followed by Susskind and Glogower formalism based on Sine and Cosine operators [29]. An important contribution to this problem is the Barnett-Pegg formalism [31] which is used in this work. In what follows, we will also briefly introduce notions, such as quantum phase distribution, angular $Q$ phase function, phase fluctuation parameters, phase dispersion, quantum phase estimation to study the phase properties of the quantum states of our interest.

\section{A. Quantum phase distribution}

A distribution function allows us to calculate expectation values of an operator analogous to that from the corresponding density matrix. Phase distribution function for a given density operator [43, 50] can be defined as

$$
P_{\theta}=\frac{1}{2 \pi}\langle\theta|\varrho| \theta\rangle
$$

where the phase state $|\theta\rangle$, complementary to the number state $|n\rangle$, is defined [43] as 


$$
|\theta\rangle=\sum_{n=0}^{\infty} e^{\iota n \theta}|n\rangle .
$$

From the definition of the phase distribution (3), it can be observed that for a Fock state, $P_{\theta}=\frac{1}{2 \pi}$, implying it has a uniform distribution of phase. Interestingly, the states of our interest, PADFS and PSDFS, are obtained by displacing the Fock state followed by photon addition/subtraction. Therefore, we will study here what is the effect of application of displacement operator on a uniformly phase distributed (Fock) state and how subsequent photon addition/subtraction further alters the phase distribution. Using phase distribution function, the information regarding uncertainty in phase and phase fluctuation can also be obtained.

\section{B. Angular $Q$ phase function}

Analogous to the phase distribution $P_{\theta}$, phase distributions are also defined as radius integrated quasidistribution functions which are used as the witnesses for quantumness [4]. One such phase distribution function based on the angular part of the $Q$ function is studied in $[45,46]$. Specifically, the angular $Q$ function is defined as

$$
Q_{\theta_{1}}=\int_{0}^{\infty} Q\left(\beta, \beta^{\star}\right)|\beta| d|\beta|,
$$

where the $Q$ function [75] is defined as the projection of the state of interest on the coherent state basis, i.e.,

$$
Q=\frac{1}{\pi}\langle\beta|\rho| \beta\rangle
$$

with coherent state parameter $\beta=|\beta| \exp \left[\iota \theta_{1}\right]$. The relevance of the $Q$ function as witness of nonclassicality [4] and in state tomography [76] is well studied. On top of that, non-Gaussianity of the PADFS and PSDFS using $Q$ function was recently reported by us [20].

\section{Quantum phase fluctuation}

In attempts to get rid of the limitations of the Hermitian phase operator of Dirac [28], Louisell [74] first mentioned that bare phase operator should be replaced by periodic functions. As a consequence, sine $(\hat{\mathcal{S}})$ and cosine $(\hat{\mathcal{C}})$ operators appeared, explicit forms of these operators were given by Susskind and Glogower [29], and further modified by Barnett and Pegg [31] as

$$
\hat{\mathcal{S}}=\frac{\hat{a}-\hat{a}^{\dagger}}{2 \iota\left(\bar{N}+\frac{1}{2}\right)^{\frac{1}{2}}}
$$

and

$$
\hat{\mathcal{C}}=\frac{\hat{a}+\hat{a}^{\dagger}}{2\left(\bar{N}+\frac{1}{2}\right)^{\frac{1}{2}}} .
$$

Here, $\bar{N}$ is the average number of photons in the measured field, and here we refrain our discussion to Barnett and Pegg sine and cosine operators [31]. Carruthers and Nieto [26] have introduced three quantum phase fluctuation parameters in terms of sine and cosine operators

$$
\begin{gathered}
U=(\Delta N)^{2}\left[(\Delta \mathcal{S})^{2}+(\Delta \mathcal{C})^{2}\right] /\left[\langle\hat{\mathcal{S}}\rangle^{2}+\langle\hat{\mathcal{C}}\rangle^{2}\right] \\
S=(\Delta N)^{2}(\Delta \mathcal{S})^{2}
\end{gathered}
$$


and

$$
Q=S /\langle\hat{\mathcal{C}}\rangle^{2}
$$

Note that Carruthers and Nieto [26] had introduced these parameters in terms of Susskind and Glogower operators [29]; here we use them in Barnett-Pegg formalism to remain consistent with [77], where $U$ parameter is shown relevant as a witness of nonclassicality [77]. Specifically, $U$ is 0.5 for coherent state, and reduction of $U$ parameter below the value for coherent state can be interpreted as the presence of nonclassical behavior [77]. In what follows, we will study quantum phase fluctuations for PADFS and PSDFS by computing analytic expressions of $U, S$ and $Q$ parameters in Barnett-Pegg formalism, with a specific focus on the possibility of witnessing nonclassical properties of these states via the reduction of $U$ parameter below the coherent state limit.

\section{Phase dispersion}

A known application of phase distribution function (3) is that it can be used to quantify the quantum phase fluctuation. Although the variance is also used occasionally as a measure of phase fluctuation, it has a drawback that it depends on the origin of phase integration [50]. A measure of phase fluctuation, free from this problem, is phase dispersion [25] defined as

$$
D=1-\left|\int_{-\pi}^{\pi} d \theta \exp [-\iota \theta] P_{\theta}\right|^{2} .
$$

Here, it is worth stressing that both Carruthers-Nieto parameters and phase dispersion $D$ correspond to phase fluctuation, Our primary focus is to study phase fluctuation and further to check the correlation between these measures of phase fluctuation. Thus, it would be interesting to study phase fluctuation from the two perspectives.

\section{E. Quantum phase estimation}

Quantum phase estimation is performed by sending the input state through a Mach-Zehnder interferometer and applying the phase to be determined $(\phi)$ on one of the arms of the interferometer. To study the phase estimation using Mach-Zehnder interferometer, angular momentum operators [60, 78], defined as

$$
\begin{aligned}
& \hat{J}_{x}=\frac{1}{2}\left(\hat{a}^{\dagger} \hat{b}+\hat{b}^{\dagger} \hat{a}\right), \\
& \hat{J}_{y}=\frac{\iota}{2}\left(\hat{b}^{\dagger} \hat{a}-\hat{a}^{\dagger} \hat{b}\right),
\end{aligned}
$$

and

$$
\hat{J}_{z}=\frac{1}{2}\left(\hat{a}^{\dagger} \hat{a}-\hat{b}^{\dagger} \hat{b}\right)
$$

are used. Here, $\hat{a}$ and $\hat{b}$ are the annihilation operators for the modes corresponding to two input ports of the MachZehnder interferometer. The average value of $\hat{J}_{z}$ operator in the output of the Mach-Zehnder interferometer, which is one-half of the difference of photon numbers in the two output ports (15), can be written as

$$
\left\langle\hat{J}_{z}\right\rangle=\cos \phi\left\langle\hat{J}_{z}\right\rangle_{\text {in }}-\sin \phi\left\langle\hat{J}_{x}\right\rangle_{\text {in }}
$$

Therefore, variance in the measured value of operator $\hat{J}_{z}$ can be computed as

$$
\left(\Delta J_{z}\right)^{2}=\cos ^{2} \phi\left(\Delta J_{z}\right)_{i n}^{2}+\sin ^{2} \phi\left(\Delta J_{x}\right)_{i n}^{2}-2 \sin \phi \cos \phi \operatorname{cov}\left(\hat{J}_{x}, \hat{J}_{z}\right)_{i n},
$$


where covariance of the two observables is defined as

$$
\operatorname{cov}\left(\hat{J}_{x}, \hat{J}_{z}\right)=\frac{1}{2}\left\langle\hat{J}_{x} \hat{J}_{z}+\hat{J}_{z} \hat{J}_{x}\right\rangle-\left\langle\hat{J}_{x}\right\rangle\left\langle\hat{J}_{z}\right\rangle
$$

This allows us to quantify precision in phase estimation [78] as

$$
\Delta \phi=\frac{\Delta J_{z}}{\left|\frac{d\left\langle\hat{J}_{z}\right\rangle}{d \phi}\right|}
$$

In what follows, we have also computed and analyzed $\Delta \phi$ for the quantum states of our interest.

In this section, we have introduced a number of interesting parameters/measures that can be used to study the phase properties of a quantum state. In the next section, we will briefly introduce the quantum states of our interest and in the subsequent section, we aim to study their phase properties.

\section{QUANTUM STATES OF OUR INTEREST}

We have already mentioned that our focus would be on PADFS and PSDFS. In the Fock basis, a DFS may be described as [16]

$$
|\Phi(n, \alpha)\rangle=\hat{D}(\alpha)|n\rangle=\frac{N}{\sqrt{n !}} \sum_{p=0}^{n}\left(\begin{array}{l}
n \\
p
\end{array}\right)\left(-\alpha^{\star}\right)^{(n-p)} \exp \left[-\frac{|\alpha|^{2}}{2}\right] \sum_{m=0}^{\infty} \frac{\alpha^{m}}{m !} \sqrt{(m+p) !}|m+p\rangle
$$

where the displacement operator $\hat{D}(\alpha)=\exp \left(\alpha \hat{a}^{\dagger}-\alpha^{\star} \hat{a}\right)$ is defined in terms of displacement parameter $\alpha=$ $|\alpha| \exp \left(\iota \theta_{2}\right)$, and $|n\rangle$ is the initial Fock state chosen to be displaced with $n$ being the Fock parameter. Using this state, $u$-PADFS can be defined as [20]

$$
\left.\left|\psi_{+}(u, n, \alpha)\right\rangle=N_{+} \hat{a}^{\dagger u}|\Phi(n, \alpha)\rangle=\frac{N_{+}}{\sqrt{n !}} \sum_{p=0}^{n}\left(\begin{array}{l}
n \\
p
\end{array}\right)\left(-\alpha^{\star}\right)^{(n-p)} \exp \left(-\frac{|\alpha|^{2}}{2}\right) \sum_{m=0}^{\infty} \frac{\alpha^{m}}{m !} \sqrt{(m+p+u) !} m+p+u\right\rangle
$$

Similarly, we can define $v$-PSDFS as [20]

$$
\left|\psi_{-}(v, n, \alpha)\right\rangle=N_{-} \hat{a}^{v}|\Phi(n, \alpha)\rangle=\frac{N_{-}}{\sqrt{n !}} \sum_{p=0}^{n}\left(\begin{array}{l}
n \\
p
\end{array}\right)\left(-\alpha^{\star}\right)^{(n-p)} \exp \left(-\frac{|\alpha|^{2}}{2}\right) \sum_{m=0}^{\infty} \frac{\alpha^{m}}{m !} \frac{(m+p) !}{\sqrt{(m+p-v) !}}|m+p-v\rangle .
$$

Here, $m$ and $p$ are the real integers. Further, normalization constants for PADFS and PSDFS can be obtained as

$$
N_{+}=\left[\frac{1}{n !} \sum_{p, p^{\prime}=0}^{n}\left(\begin{array}{l}
n \\
p
\end{array}\right)\left(\begin{array}{c}
n \\
p^{\prime}
\end{array}\right)\left(-\alpha^{\star}\right)^{(n-p)}(-\alpha)^{\left(n-p^{\prime}\right)} \exp \left[-|\alpha|^{2}\right] \sum_{m=0}^{\infty} \frac{\alpha^{m}\left(\alpha^{\star}\right)^{m+p-p^{\prime}}(m+p+u) !}{m !\left(m+p-p^{\prime}\right) !}\right]^{-0.5}
$$

and

$$
N_{-}=\left[\frac{1}{n !} \sum_{p, p^{\prime}=0}^{n}\left(\begin{array}{c}
n \\
p
\end{array}\right)\left(\begin{array}{c}
n \\
p^{\prime}
\end{array}\right)\left(\alpha^{\star}\right)^{(n-p)}(-\alpha)^{\left(n-p^{\prime}\right)} \exp \left[-|\alpha|^{2}\right] \sum_{m=0}^{\infty} \frac{\alpha^{m}\left(\alpha^{\star}\right)^{m+p-p^{\prime}}(m+p) !}{m !\left(m+p-p^{\prime}\right) !(m+p-v) !}\right]^{-0.5},
$$

respectively. Here, the subscripts + and - correspond to the photon addition and subtraction, respectively. A schematic scheme for generation of PADFS/PSDFS from DFS has been proposed recently by us (see Fig. 1 in Ref. [20]). Due to the general form of PADFS and PSDFS, a large number of states can be obtained in the limiting cases. Some of the important limiting cases of PADFS and PSDFS in the present notation are summarized in Table I. This table clearly establishes that the applicability of the results obtained in the present study is not restricted to PADFS and PSDFS; rather an investigation of the phase properties of PADFS and PSDFS would also reveal phase properties of many other quantum states of particular interest. 


\begin{tabular}{cccc}
\hline Reduction of state & Name of the state & Reduction of state & Name of the state \\
\hline \hline$\left|\psi_{+}(u, n, \alpha)\right\rangle$ & $u$-PADFS & $\left|\psi_{-}(v, n, \alpha)\right\rangle$ & $v$-PSDFS \\
$\left.-\psi_{+}(0, n, \alpha)\right\rangle$ & DFS & $\left.-\psi_{-}(0, n, \alpha)\right\rangle$ & DFS \\
$\left|\psi_{+}(0,0, \alpha)\right\rangle$ & Coherent state & $\left|\psi_{-}(0,0, \alpha)\right\rangle$ & Coherent state \\
$\left.-\psi_{+}(0, n, 0)\right\rangle$ & Fock state & $\left.-\psi_{-}(0, n, 0)\right\rangle$ & Fock state \\
$\left.-\psi_{+}(u, 0, \alpha)\right\rangle$ & $u$-Photon added & $\left.-\psi_{-}(v, 0, \alpha)\right\rangle$ & $v$-Photon subtracted \\
& coherent state & & coherent state \\
\hline
\end{tabular}

TABLE I. Various states that can be obtained as the limiting cases of the PADFS and PSDFS.

\section{PHASE PROPERTIES OF PADFS AND PSDFS}

The description of the states of our interest given in the previous section can be used to study different phase properties and quantify phase fluctuation in the set of quantum states listed in Table I. Specifically, with the help of the quantum states defined in Eqs. (21)-(22), we have obtained the analytic expressions of phase distribution and other phase parameters defined in Section II.

\section{A. Phase distribution function}

To begin with, we compute the analytic expressions of $P_{\theta}$ for the PADFS and PSDFS, using Eq. (3) as

$$
\begin{aligned}
P_{\theta}(u, n) & =\frac{1}{2 \pi} \frac{\left|N_{+}\right|^{2}}{n !} \sum_{p, p^{\prime}=0}^{n}\left(\begin{array}{c}
n \\
p
\end{array}\right)\left(\begin{array}{c}
n \\
p^{\prime}
\end{array}\right) \exp \left[-|\alpha|^{2}\right]|\alpha|^{2 n-p-p^{\prime}} \\
& \times \sum_{m, m^{\prime}=0}^{\infty} \frac{(-|\alpha|)^{m+m^{\prime}} \sqrt{(m+p+u) !\left(m^{\prime}+p^{\prime}+u\right) !}}{m ! m^{\prime} !} \exp \left[\iota\left(\theta-\theta_{2}\right)\left(m^{\prime}+p^{\prime}-m-p\right)\right]
\end{aligned}
$$

and

$$
\begin{aligned}
P_{\theta}(v, n) & =\frac{1}{2 \pi} \frac{\left|N_{-}\right|^{2}}{n !} \sum_{p, p^{\prime}=0}^{n}\left(\begin{array}{c}
n \\
p
\end{array}\right)\left(\begin{array}{c}
n \\
p^{\prime}
\end{array}\right) \exp \left[-|\alpha|^{2}\right]|\alpha|^{2 n-p-p^{\prime}} \\
& \times \sum_{m, m^{\prime}=0}^{\infty} \frac{(-|\alpha|)^{m+m^{\prime}}(m+p) !\left(m^{\prime}+p^{\prime}\right) !}{m ! m^{\prime} ! \sqrt{(m+p-v) !\left(m^{\prime}+p^{\prime}-v\right) !}} \exp \left[\iota\left(\theta-\theta_{2}\right)\left(m^{\prime}+p^{\prime}-m-p\right)\right],
\end{aligned}
$$

respectively. Since the obtained expressions in Eqs. (23) and (24) are complex in nature, we depict numerical (graphical) analysis of the obtained results in Figs. 1 and 2 for PADFS and PSDFS, respectively. Specifically, in Fig. 1 (a), we have shown the variation of phase distribution with phase parameter $\theta$ for different number of photon added in the displaced single photon Fock state $(D(\alpha)|1\rangle)$ for $\theta_{2}=0$. A uniform phase distribution for Fock state (with a constant value of $\frac{1}{2 \pi}$ ) is found to transform to one that decreases for higher values of phase and possess a dip in the phase distribution for $\theta=0$, which can be thought of as an approach to the Fock state. In fact, in case of classical states, $P_{\theta}$ has a peak at zero phase difference $\theta-\theta_{2}$, and therefore, this contrasting behavior can be viewed as signature of quantumness of DFS. However, with the increase in the number of photons added to the DFS, the phase distribution of the PADFS is observed to become narrower. In fact, a similar behavior with increase in the mean photon number of coherent state was observed previously [43]. It is imperative to state that $P_{\theta}$ in case of higher number of photon added to DFS has similar but narrower distribution than that of coherent state. In contrast, with increase in the Fock parameter, the phase distribution is observed to become broader (cf. Fig. 1 (b)). Thus, the increase in the number of photons added and the increase in Fock parameter have opposite effects on the phase distribution. The same is also illustrated through the polar plots in Fig. 1 (c)-(d), which not only reestablish the same fact, but also illustrate the dependence of $P_{\theta}$ on the phase of the displacement parameter. Specifically, the obtained phase distribution remains symmetric along the value of phase $\theta_{2}$ (i.e., $P_{\theta}$ is observed to have a mirror symmetry along $\theta=\theta_{2}$ ) of the displacement parameter. The phase distribution of Fock state is shown by a black circle in the polar plot.

Instead of photon addition, if we subtract photons from the DFS, a similar effect on the phase distribution to that of photon addition is observed. Further, a comparison between photon addition and subtraction on the phase distribution establishes that a single photon subtraction has a prominent impact on phase distribution when compared to that of single photon addition, i.e., the distribution can be observed to be narrower than that of coherent state in most of 


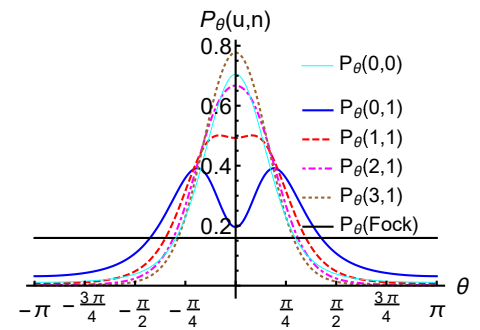

(a)

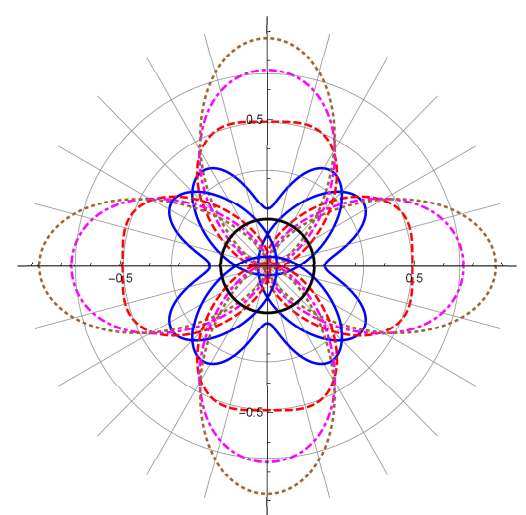

(c)

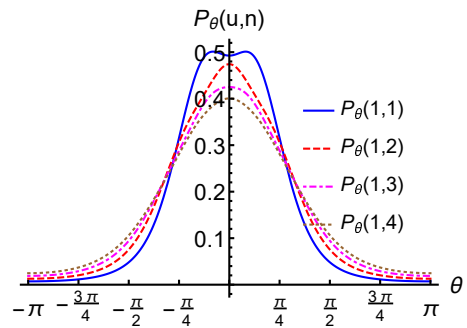

(b)

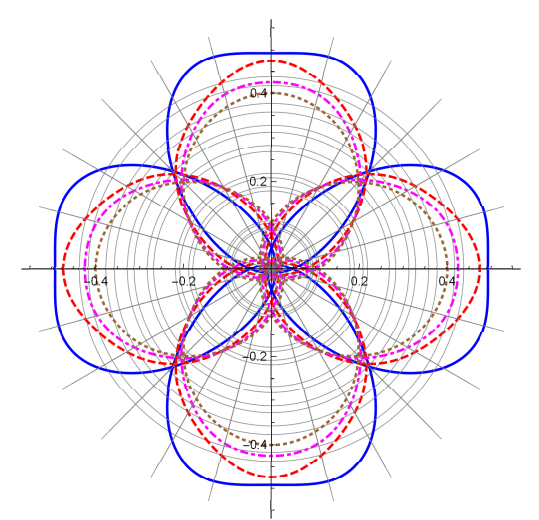

(d)

FIG. 1. (Color online) Variation of phase distribution function with phase parameter for PADFS with displacement parameter $|\alpha|=1$ for different values of photon addition ((a) and (c)) and Fock parameters ((b) and (d)). The phase distribution is shown using both two-dimensional ((a) and (b) with $\left.\theta_{2}=0\right)$ and polar ((c) and (d)) plots. In (c) and (d), $\theta_{2}=\frac{n \pi}{2}$ with integer $n \in[0,3]$, and the legends are same as in (a) and (b), respectively.

the cases for $u=v$. For instance, single photon added (subtracted) DFS is broader (narrower) than corresponding coherent state. Similarly, with the increase in the value of Fock parameter, we can observe more changes on PSDFS than what was observed in PADFS, i.e., the phase distribution broadens more with Fock parameter for PSDFS. Note that $P_{\theta}$ has a peak at $\theta=\theta_{2}$ only for photon addition $u>n$, while in case of photon subtraction it can be observed for $v \geq n$. With the increase in the amplitude of displacement parameter $(|\alpha|)$ initially the phase distribution becomes narrower, which is further supported by both addition and subtraction of photons, but it becomes broader again for very high $|\alpha|$ (figure is not shown here).

\section{B. Angular $Q$ function of PADFS and PSDFS}

We further discuss a phase distribution based on $Q$ function using Eq. (5). In this particular case, we have obtained the angular $Q$ function from the $Q$ functions of the PADFS and PSDFS reported as Eqs. (15)-(16) in [20]. Specifically, we have shown the effect of photon addition on the DFS $(D(\alpha)|1\rangle)$ for a specific value of the displacement parameter in Fig. 3 (a) for angular $Q$ function. One can clearly see that the polar plots show an increase in the peak (located at $\theta_{1}=\theta_{2}$ ) of the distribution with photon addition. Further, one can compare the behavior of $Q_{\theta_{1}}$ with $P_{\theta}$ in Fig. 1 and observe that they behave quite differently (as reported in [43] for the coherent states), other than increase in the peak of the distribution. Specifically, $P_{\theta}$ has a peak at $\theta=\theta_{2}$ only for $u>n$, while $Q_{\theta_{1}}$ is always peaked at the phase of the displacement parameter which also becomes a line of symmetry. Interestingly, the effect of increase in the Fock parameter of PADFS on $Q_{\theta_{1}}$ is similar but less prominent in comparison to photon addition. This is in quite contrast of that observed for $P_{\theta}$ (in Figs. 1 and $3(\mathrm{~b})$ ). In case of PSDFS, both photon subtraction and Fock parameter have completely different effects on $Q_{\theta_{1}}$ (cf. Fig. $3(\mathrm{c})$-(d)) which is also in contrast to that on corresponding $P_{\theta}$ (shown in Fig. 2). Specifically, with increase in photon subtraction the angular $Q$ function becomes narrower peaked at $\theta=\theta_{2}$, but for larger number of photon subtraction the peak value decreases quickly. However, with increasing Fock parameter (cf. Fig. $3(\mathrm{~d})), Q_{\theta_{1}}$ behaves much like photon addition on DFS (shown in Fig. 3 (a)). The observed behavior shows the relevance of studying both these phase distributions due to their independent characteristics. 


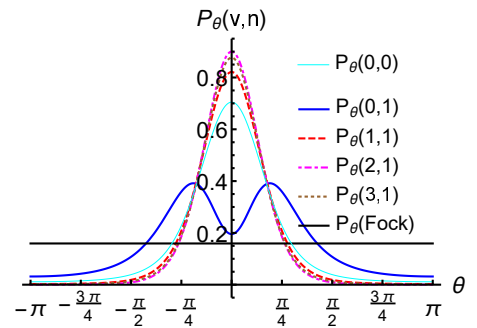

(a)

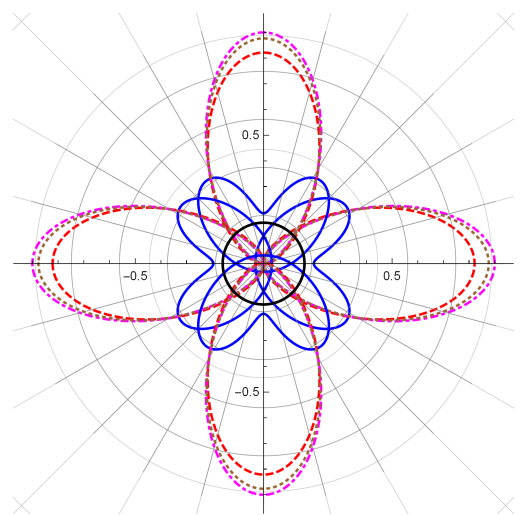

(c)

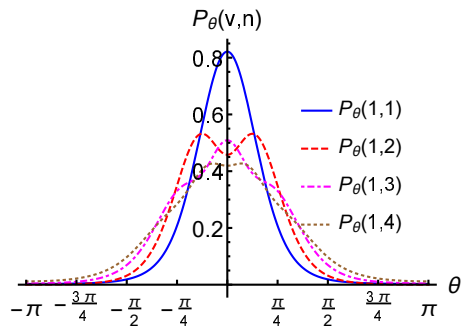

(b)

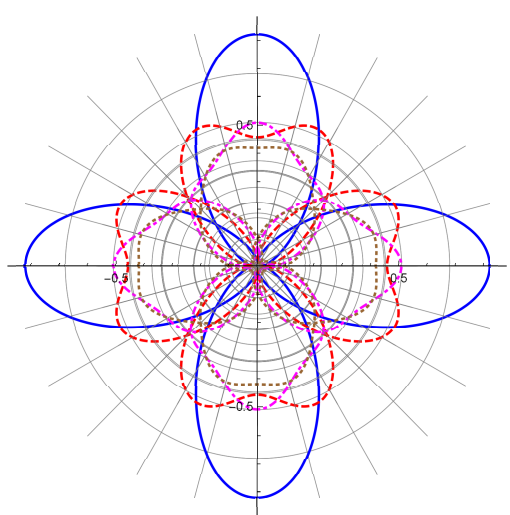

(d)

FIG. 2. (Color online) Variation of phase distribution function with phase parameter for PSDFS with displacement parameter $|\alpha|=1$ for different values of photon subtraction ((a) and (c)) and Fock parameters ((b) and (d)). The phase distribution is shown using both two-dimensional ((a) and (b) with $\left.\theta_{2}=0\right)$ and polar ((c) and (d)) plots. In (c) and (d), $\theta_{2}=\frac{n \pi}{2}$ with integer $n \in[0,3]$, and the legends are same as in (a) and (b), respectively.

\section{Quantum phase fluctuation of PADFS and PSDFS}

Carruthers and Nieto [26] introduced three parameters to study quantum phase fluctuation (9)-(11). It was only in the recent past that some of the present authors provided a physical meaning to one of these parameters by establishing its relation with antibunching and sub-Poissonian photon statistics. Thus, the quantum phase fluctuation studied here using three parameters will also be used to witness the nonclassical nature of the quantum states under consideration. Here, the effect of photon addition/subtraction and displacement parameters on these fluctuation parameters is also studied (shown in Fig. 4). Specifically, Fig. 4 (a)-(c) show variation of the three parameters of quantum phase fluctuation for different values of the number of photons added in the displaced Fock state $(D(\alpha)|1\rangle)$ with displacement parameter $|\alpha|$. It may be clearly observed that two of the quantum phase fluctuation parameters, namely $U(u, n)$ and $Q(u, n)$ decrease with the value of displacement parameter, while $S(u, n)$ increases with $|\alpha|$. Interestingly, the photon addition and increase in the displacement parameter exhibit the same effect on all three quantum phase fluctuation parameters for PADFS, while for higher values of displacement parameter $S(u, n)$ show completely opposite effect of photon addition. In contrast, $U(v, n)$ for $v$ subtracted photons from $D(\alpha)|1\rangle$ is found to increase (decrease) with photon subtraction while decrease (increase) with the displacement parameter for small (large) value of $|\alpha|$ (cf. Fig. 4 (d)). On the other hand, parameter $S(v, n)$ is also observed to increase (decrease) with $|\alpha|(v)$ as shown in Fig. $4(\mathrm{e})$. The third parameter $Q(v, n)$ shows slightly complex behavior for PSDFS with both $|\alpha|$ and $v$ (cf. Fig. 4 (f)) as it behaves analogous to PADFS for each subtracted photon for both small and large values of the displacement parameter (when it increases with $|\alpha|$ ), but for intermediate values the behavior is found to be completely opposite.

As mentioned previously, $U(i, n) \forall i \in\{u, v\}$ has a physical significance as a witness of antibunching for values of this parameter less than $\frac{1}{2}$, Fig. 4 (a) and (d) can be used to perform similar studies for PADFS and PSDFS, respectively. In case of PADFS, we can observe this relevant parameter to become less than $\frac{1}{2}$, and thus to illustrate the presence of antibunching, only at higher values of the displacement parameter and photon added to the displaced Fock state. In contrast, PSDFS shows the presence of this nonclassical feature in all cases.Thus, occurrence of antibunching in PADFS and PSDFS is established here through this phase fluctuation parameter. Interestingly, a similar dependence of antibunching in PADFS and PSDFS has been recently reported by us [20] using a different 


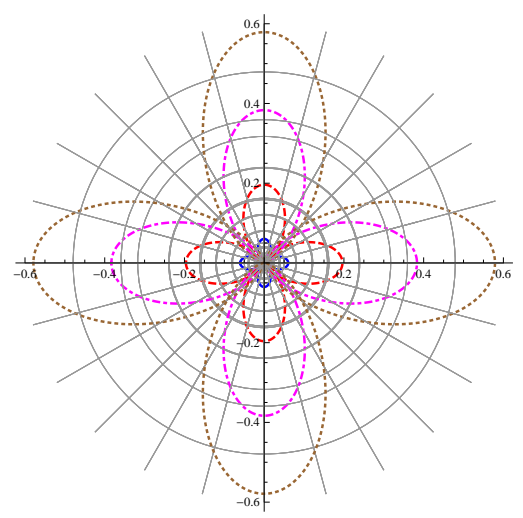

(a)

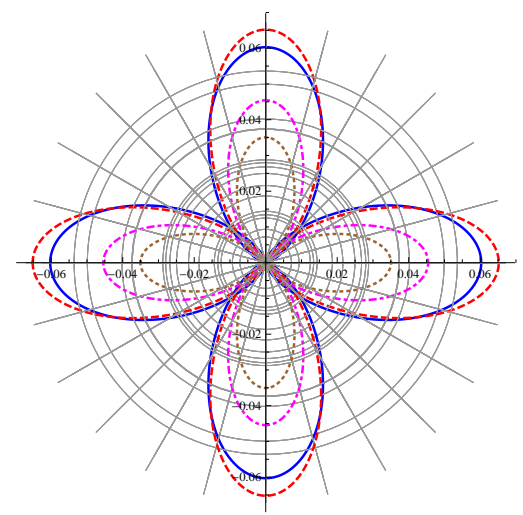

(c)

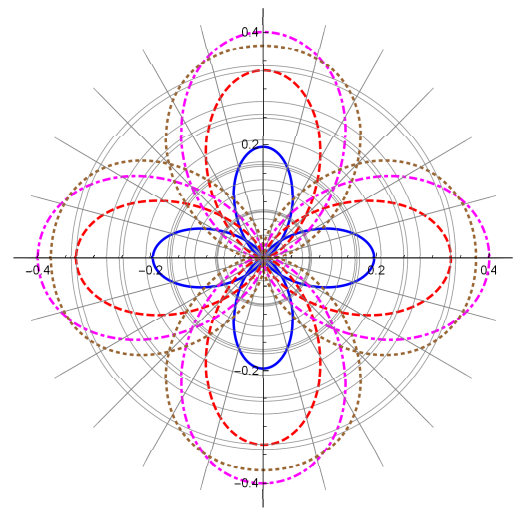

(b)

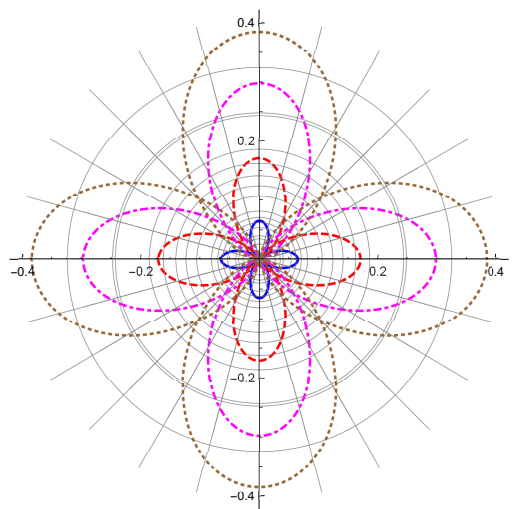

(d)

FIG. 3. (Color online) The polar plots for angular $Q$ function for PADFS (in (a) and (b)) and PSDFS (in (c) and (d)) for displacement parameter $|\alpha|=1$ and $\theta_{2}=\frac{n \pi}{2}$ with integer $n \in[0,3]$ for different values of photon addition/subtraction and Fock parameters. In (a) and (c), for $n=1$, the smooth (blue), dashed (red), dot-dashed (magenta), and dotted (brown) lines correspond to photon addition/subtraction $0,1,2$, and 3, respectively. In (b) and (d), for the single photon added/subtracted displaced Fock state, the smooth (blue), dashed (red), dot-dashed (magenta), and dotted (brown) lines correspond to Fock parameter $1,2,3$, and 4 , respectively.

criterion. Further, one can observe from the expression of $U$ in Eq. (9) that it is expected to be independent of the phase of the displacement parameter, which can also be understood from the use of this parameter as a witness for an intensity moments based nonclassical feature. In contrast, $S$ and $Q$ in Eqs. (10)-(11) show dependence on the phase of displacement parameter. Here, we have not discussed the effect of Fock parameter in detail, but in case of photon addition, $u$ and $n$ have same (opposite) effects on $S(U$ and $Q)$ parameter(s). Fock parameter has always shown opposite effect of photon subtraction on all three phase fluctuation parameters, and thus nonclassicality revealed by $U$ can be enhanced with Fock parameter. The relevance of Fock parameter can also be visualized by observing the fact that the single photon subtracted coherent state has $U=0.5$ (which is consistent with the value zero of the antibunching witness reported in [79]). Thus, in this case, the origin of the induced antibunching can be attributed to the non-zero value of Fock parameter.

\section{Phase Dispersion}

We compute a measure of quantum phase fluctuation based on quantum phase distribution, the phase dispersion (12), for both PADFS and PSDFS to perform a comparative study between them. Specifically, the maximum value of dispersion is 1 which corresponds to the uniform phase distribution, i.e., $P_{\theta}=\frac{1}{2 \pi}$. Both PADFS and PSDFS show a uniform distribution for the displacement parameter $\alpha=0$ (cf. Fig. 5). It is a justified result as both the states reduce to the Fock state in this case. However, with the increase in the value of displacement parameter quantum phase dispersion is found to decrease. This may be attributed to the number-phase complimentarity [80-82], which leads to smaller phase fluctuation with increasing variance in the number operator at higher values of displacement parameter. 


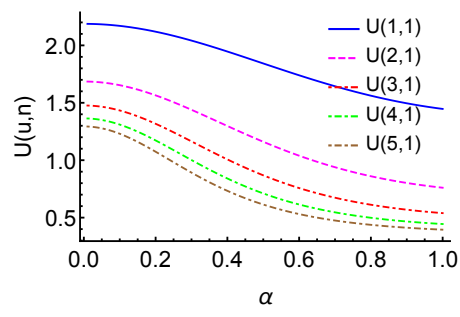

(a)

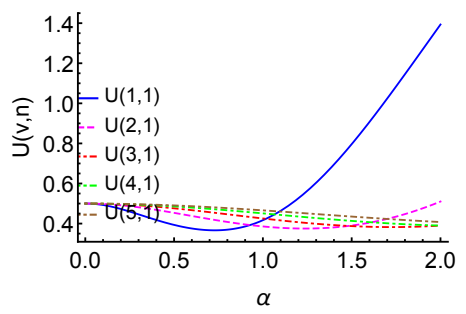

(d)

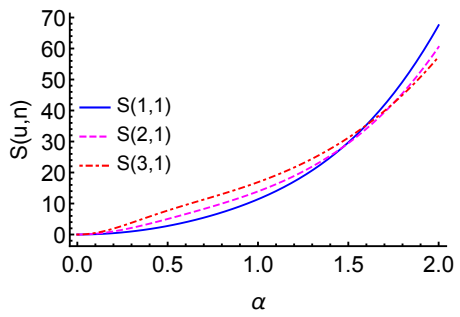

(b)

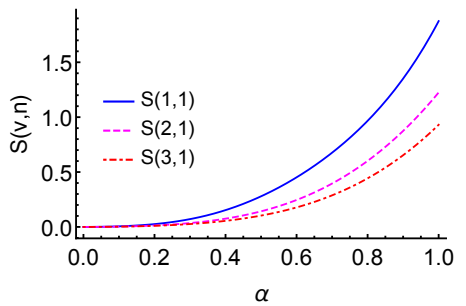

(e)

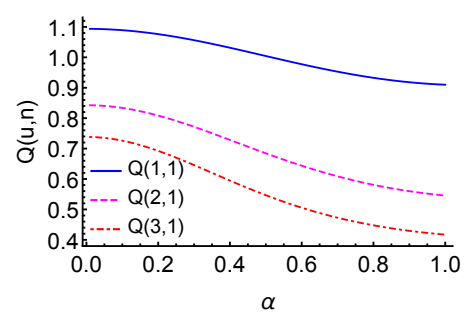

(c)

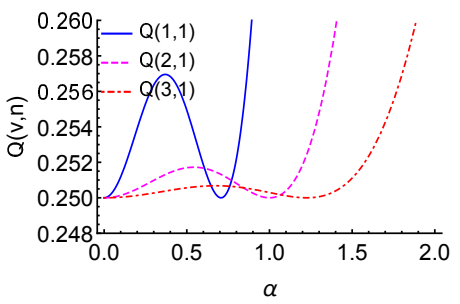

(f)

FIG. 4. (Color online) Variation of three phase fluctuation parameters introduced by Carruthers and Nieto with the displacement parameter with $\theta_{2}=0$. The values of photon addition $(u)$, subtraction $(v)$, and Fock parameter $n=1$ are given in the legends. Parameter $U(i, n) \forall i \in\{u, v\}$ also illustrates antibunching in the states for values less than $\frac{1}{2}$.

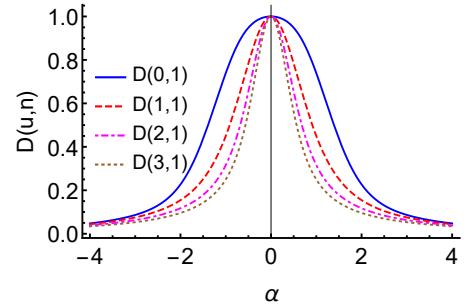

(a)

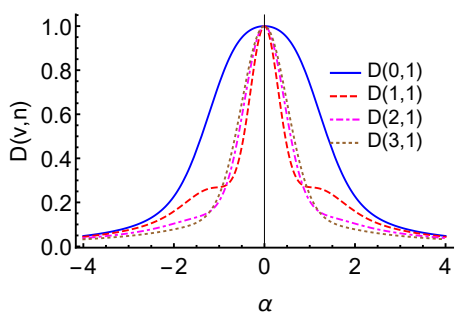

(c)

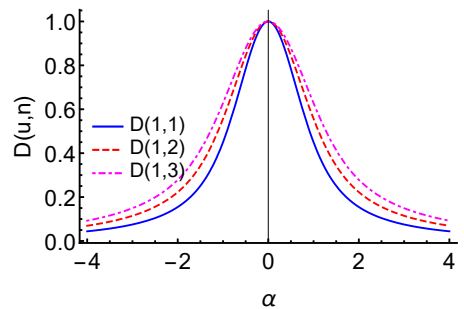

(b)

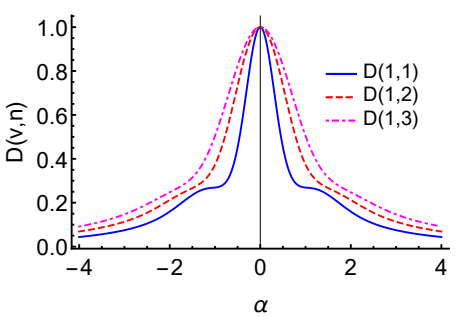

(d)

FIG. 5. (Color online) Variation of phase dispersion for PADFS (in (a) and (b)) and PSDFS (in (c) and (d)) with displacement parameter for an arbitrary $\theta_{2}$. Dependence on different values of photon added/subtracted and the initial Fock state $|1\rangle$ (in (a) and (c)), while on different values of Fock parameter for single photon added/subtracted state (in (b) and (d)).

Thus, with an increase in the average photon number by increasing the displacement parameter, phase dispersion decreases for both PADFS and PSDFS. Addition of photons in DFS leads to decrease in the value of phase dispersion, while subtraction of photons has more complex effect on phase dispersion (cf. Fig. 5 (a) and (c)). Specifically, for the smaller values of the displacement parameter $(|\alpha|<1)$, the phase dispersion parameter behaves differently for $v \leq n$ and $v>n$. This can be attributed to the sub-Poissonian photon statistics for $v \leq n$ with $|\alpha|<1$ as well as the small value of average photon number (Fig. 4 (d)). However, at the higher values of the displacement parameter $D$ for the PSDFS behaves in a manner analogous to the PADFS. Interestingly, increase in the Fock parameter shows similar effect on PADFS and PSDFS in Fig. 5 (b) and (d), respectively. 


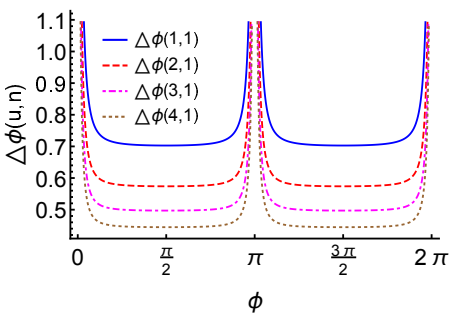

(a)

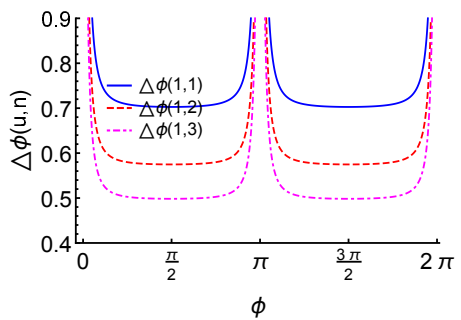

(c)

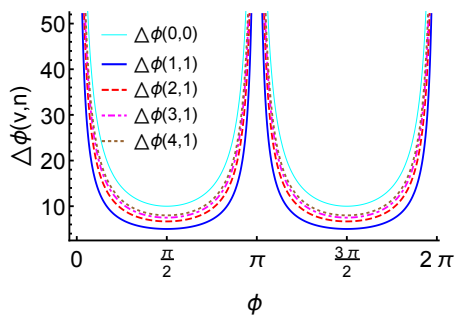

(b)

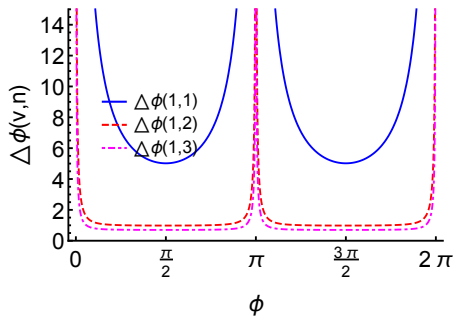

(d)

FIG. 6. Phase sensing uncertainty for (a) PADFS and (b) PSDFS as a function of phase to be estimated $\phi$ for different number of photon addition/subtraction with $n=1$. The dependence for (c) PADFS and (d) PSDFS is also shown for different values of Fock parameters with $u=1$ and $v=1$, respectively. In all cases, we have chosen $\alpha=0.1$.

\section{E. Phase sensing uncertainity for PADFS and PSDFS}

We finally discuss quantum phase estimation using Eq. (19), assuming the two mode input state in the MachZehnder interferometer as $\left|\psi_{i}(j, n, \alpha)\right\rangle \otimes|0\rangle$. The expressions for the variance of the difference in the photon numbers in the two output modes of the Mach-Zehnder interferometer for input PADFS and PSDFS and the rest of the parameters required to study phase sensing are reported in Appendix.

The obtained expressions allow us to study the optimum choice of state parameters for quantum phase estimation using PADFS and PSDFS. The variation of these parameters is shown in Fig. 6. Specifically, we have shown that PSDFS is preferable over coherent state for phase estimation (cf. Fig. 6 (b)). However, with the increase in the photon subtraction this phase uncertainty parameter is found to increase although remaining less than corresponding coherent state value. In contrast, with photon addition, advantage in phase estimation can be attained as the reduction of the phase uncertainty parameter allows one to perform more precise measurement. This advantage can be enhanced further by choosing large values of photon addition and Fock parameter (cf. Fig. 6 (a) and (c)). In a similar sense, appropriate choice of Fock parameter would also be advantageous in phase estimation with PSDFS as it decreases the phase uncertainty parameter, but still PADFS remains preferable over PSDFS. This can further be controlled by an increase in $|\alpha|$ which decreases (increases) phase uncertainty parameter for PADFS (PSDFS).

\section{CONCLUSIONS}

A set of engineered quantum states can be obtained as the limiting cases from the PADFS and PSDFS, i.e., DFS, coherent state, photon added/subtracted coherent state, and Fock state. Specifically, PADFS/PSDFS are obtained by application of unitary (displacement) and non-unitary (addition and subtraction of photons) operations on Fock state. In view of the fact that the Fock states have uniform phase distribution, the set of unitary and non-unitary quantum state engineering operations are expected to affect the phase properties of the generated state. Therefore, here we have calculated quantum phase distribution, which further helped in quantifying phase fluctuation as phase dispersion. We have also computed the phase distribution as the angular $Q$ function. We have further studied phase fluctuation using three Carruthers and Nieto parameters, and have used one of them to reveal the existence of antibunching in the quantum states of our interest.

Both the phase distribution and angular $Q$ functions are found to be symmetric along the value of the phase of the displacement parameter. The phase distribution is observed to become narrow and peak(s) to increase with the amplitude of the displacement parameter $(|\alpha|)$, which further becomes broader for higher values of $|\alpha|$. Further, photon addition/subtraction and Fock parameters are observed to have opposite effects on phase distribution, i.e., distribution 
function becomes narrower (broader) with photon addition/subtraction (Fock parameter). Among photon addition and subtraction operations, subtracting a photon alters the phase properties more than that of photon addition. Specifically, at the small values of the displacement parameter $(|\alpha|<1)$, the phase properties of PSDFS for $v \leq n$ and $v>n$ behave differently. This can be attributed to the fact that for $v \leq n$ with $|\alpha|<1$, the average photon number becomes very small. Further, the peak of the phase distribution remains at the phase of displacement parameter only when the number of photons added/subtracted is more than that of the Fock parameter. However, in case the number of photons subtracted (added) is same as the Fock parameter, the peak of the phase distribution is observed (not observed) at the phase of displacement parameter. The angular $Q$ function can be observed to show similar dependence on various parameters, but the peak of the distribution remains located at the value of phase of the displacement parameter. The three phase fluctuation parameters introduced by Carruthers and Nieto [26] show phase properties of PADFS and PSDFS, while one of them, $U$ parameter also reveals antibunching in both PADFS and PSDFS. In this case, the role of Fock parameter as antibunching inducing operation in PSDFS is also discussed. Phase dispersion quantifying phase fluctuation remains unity for Fock state reflecting uniform distribution, which can be observed to decrease with increasing displacement parameter. This may be attributed to the number-phase complimentarity as the higher values of variance with increasing displacement parameter lead to smaller phase fluctuation. Fock parameter and photon addition/subtraction show opposite effects on the phase dispersion as it increases (decreases) with $n(u / v)$.

Finally, we have also discussed the advantage of the PADFS and PSDFS in quantum phase estimation and obtained the set of optimized parameters in the PADFS/PSDFS. Both photon addition and Fock parameter decrease the uncertainty in phase estimation, while photon subtraction, though performs better than coherent state is not as advantageous as $u$ or $n$. In [83], it was established that signal-to-noise ratio is significant only when the phase shift to measure is of the same order as multiplicative inverse of the average photon number. Therefore, in case of PADFS this limitation of quantum measurement is expected to play an important role. Thus, we have shown here that state engineering tools can be used efficiently to control the phase properties of the designed quantum states for suitable applications. The study can be performed for other such operations, like squeezing, photon addition followed by subtraction or vice versa.

We conclude the paper by noting that the method used for the present study is quite general and can be applied to study phase properties of many other engineered quantum states, and a major part of the results presented here can be experimentally verified using the available technolgy. Consequently, this work is expected to lead to a number of further studies and thus to provide new insights into the phase properties of the quantum states.

Acknowledgment: KT acknowledges the financial support from the Operational Programme Research, Development and Education - European Regional Development Fund project no. CZ.02.1.01/0.0/0.0/16_019/0000754 of the Ministry of Education, Youth and Sports of the Czech Republic. AP and NA thank SERB, DST, India for the support provided through the project number EMR/2015/000393. SB and VN thank CSIR, New Delhi for support through the project No. 03(1369)/16/EMR-II. AP and KT also thank A Luks for his interest and technical remarks.

[1] R. J. Glauber, Physical Review 131, 2766 (1963).

[2] E. C. G. Sudarshan, Physical Review Letters 10, 277 (1963).

[3] K. Thapliyal, A. Pathak, B. Sen, and J. Peřina, Physical Review A 90, 013808 (2014).

[4] K. Thapliyal, S. Banerjee, A. Pathak, S. Omkar, and V. Ravishankar, Annals of Physics 362, 261 (2015).

[5] K. Thapliyal, A. Pathak, B. Sen, and J. Peřina, Physics Letters A 378, 3431 (2014).

[6] S. K. Giri, K. Thapliyal, B. Sen, and A. Pathak, Physica A: Statistical Mechanics and its Applications 466, 140 (2017).

[7] H.-A. Bachor and T. C. Ralph, A Guide to Experiments in Quantum Optics (Wiley-VCH, Weinheim, 2004).

[8] K. Vogel, V. Akulin, and W. Schleich, Physical Review Letters 71, 1816 (1993).

[9] J. Sperling, W. Vogel, and G. Agarwal, Physical Review A 89, 043829 (2014).

[10] A. Miranowicz and W. Leoński, Journal of Optics B: Quantum and Semiclassical Optics 6, S43 (2004).

[11] M. A. Marchiolli and W. D. José, Physica A: Statistical Mechanics and its Applications 337, 89 (2004).

[12] B. Escher, A. Avelar, T. da Rocha Filho, and B. Baseia, Physical Review A 70, 025801 (2004).

[13] M. Yukawa, K. Miyata, T. Mizuta, H. Yonezawa, P. Marek, R. Filip, and A. Furusawa, Optics Express 21, 5529 (2013).

[14] P. Marek, J. Provazník, and R. Filip, Optics Express 26, 29837 (2018).

[15] G. Agarwal and K. Tara, Physical Review A 43, 492 (1991).

[16] M. V. Satyanarayana, Physical Review D 32, 400 (1985).

[17] A. Wunsche, Quantum Optics: Journal of the European Optical Society Part B 3, 359 (1991).

[18] F. Ziesel, T. Ruster, A. Walther, H. Kaufmann, S. Dawkins, K. Singer, F. Schmidt-Kaler, and U. Poschinger, Journal of Physics B: Atomic, Molecular and Optical Physics 46, 104008 (2013).

[19] A. Zavatta, S. Viciani, and M. Bellini, Science 306, 660 (2004).

[20] P. Malpani, N. Alam, K. Thapliyal, A. Pathak, V. Narayanan, and S. Banerjee, Annalen der Physik 531, 1800318 (2019).

[21] A. I. Lvovsky, H. Hansen, T. Aichele, O. Benson, J. Mlynek, and S. Schiller, Physical Review Letters 87, 050402 (2001). 
[22] A. Lvovsky and S. Babichev, Physical Review A 66, 011801 (2002).

[23] A. Zavatta, S. Viciani, and M. Bellini, Physical Review A 72, 023820 (2005).

[24] A. Zavatta, V. Parigi, M. Kim, and M. Bellini, New Journal of Physics 10, 123006 (2008).

[25] V. Peřinová, A. Lukš, and J. Peřina, Phase in Optics (World Scientific, 1998).

[26] P. Carruthers and M. M. Nieto, Reviews of Modern Physics 40, 411 (1968).

[27] R. Lynch, JOSA B 4, 1723 (1987).

[28] P. A. M. Dirac, in Proceedings of the Royal Society of London A: Mathematical, Physical and Engineering Sciences, Vol. 114 (1927) pp. 243-265.

[29] L. Susskind and J. Glogower, Physics Physique Fizika 1, 49 (1964).

[30] D. T. Pegg and S. M. Barnett, Physical Review A 39, 1665 (1989).

[31] S. M. Barnett and D. T. Pegg, Journal of Physics A: Mathematical and General 19, 3849 (1986).

[32] D. Pegg and S. Barnett, EPL (Europhysics Letters) 6, 483 (1988).

[33] S. M. Barnett and D. Pegg, Physical Review A 41, 3427 (1990).

[34] Y. Imry, Physica 55, 344 (1971).

[35] B. Sanders, S. M. Barnett, and P. Knight, Optics Communications 58, 290 (1986).

[36] C. C. Gerry, Optics Communications 63, 278 (1987).

[37] D.-m. Yao, Physics Letters A 122, 77 (1987).

[38] J. Vaccaro and D. Pegg, Optics Communications 70, 529 (1989).

[39] A. Pathak and S. Mandal, Physics Letters A 272, 346 (2000).

[40] N. Alam and S. Mandal, Optics Communications 366, 340 (2016).

[41] M. Alam, S. Mandal, and M. R. Wahiddin, Optics Communications 398, 1 (2017).

[42] A. Verma and A. Pathak, Physics Letters A 373, 1421 (2009).

[43] G. S. Agarwal, S. Chaturvedi, K. Tara, and V. Srinivasan, Physical Review A 45, 4904 (1992).

[44] B. M. Garraway and P. L. Knight, Physical Review A 46, R5346 (1992).

[45] U. Leonhardt and H. Paul, Physical Review A 47, R2460 (1993).

[46] U. Leonhardt, J. A. Vaccaro, B. Böhmer, and H. Paul, Physical Review A 51, 84 (1995).

[47] J. W. Noh, A. Fougeres, and L. Mandel, Physical Review Letters 67, 1426 (1991).

[48] J. W. Noh, A. Fougeres, and L. Mandel, Physical Review A 45, 424 (1992).

[49] D. T. Smithey, M. Beck, J. Cooper, M. G. Raymer, and A. Faridani, Physica Scripta 1993, 35 (1993).

[50] S. Banerjee and R. Srikanth, Physical Review A 76, 062109 (2007).

[51] S. Banerjee, J. Ghosh, and R. Ghosh, Physical Review A 75, 062106 (2007).

[52] M. Abdel-Aty, A. Azzeer, and M. S. Abdalla, Physica A: Statistical Mechanics and its Applications 389, 3375 (2010).

[53] S. Banerjee, Open Quantum Systems: Dynamics of Nonclassical Evolution (Springer and Hindustan Book Agency, 2018).

[54] H. Zheng-Feng, Journal of Modern Optics 39, 1381 (1992).

[55] I. Mendas and D. Popovic, Journal of Physics A: Mathematical and General 26, 3313 (1993).

[56] T. Gantsog, R. Tanaś, and R. Zawodny, Optics Communications 82, 345 (1991).

[57] T. Gantsog, Physics Letters A 170, 249 (1992).

[58] K. J. Resch, K. L. Pregnell, R. Prevedel, A. Gilchrist, G. J. Pryde, J. L. O’Brien, and A. G. White, Physical ReviewLletters 98, 223601 (2007).

[59] M. A. Usuga, C. R. Müller, C. Wittmann, P. Marek, R. Filip, C. Marquardt, G. Leuchs, and U. L. Andersen, Nature Physics 6, 767 (2010).

[60] B. Sanders and G. Milburn, Physical Review Letters 75, 2944 (1995).

[61] B. L. Higgins, D. W. Berry, S. D. Bartlett, H. M. Wiseman, and G. J. Pryde, Nature 450, 393 (2007).

[62] V. Giovannetti, S. Lloyd, and L. Maccone, Physical Review Letters 96, 010401 (2006).

[63] V. Giovannetti, S. Lloyd, and L. Maccone, Nature Photonics 5, 222 (2011).

[64] J. Peřina and J. Křepelka, Optics Communications 437, 373 (2019).

[65] A. Pathak, Ph. D. Thesis, arXiv preprint quant-ph/0207034 (2002).

[66] F. Xu, B. Qi, X. Ma, H. Xu, H. Zheng, and H.-K. Lo, Optics Express 20, 12366 (2012).

[67] F. Raffaelli, P. Sibson, J. E. Kennard, D. H. Mahler, M. G. Thompson, and J. C. Matthews, arXiv preprint arXiv:1804.05046 (2018)

[68] P. Horak, Journal of Modern Optics 51, 1249 (2004).

[69] J. Denschlag, J. E. Simsarian, D. L. Feder, C. W. Clark, L. A. Collins, J. Cubizolles, L. Deng, E. W. Hagley, K. Helmerson, W. P. Reinhardt, et al., Science 287, 97 (2000).

[70] J. Ahn, T. Weinacht, and P. Bucksbaum, Science 287, 463 (2000).

[71] N. Gisin, G. Ribordy, W. Tittel, and H. Zbinden, Reviews of Modern Physics 74, 145 (2002).

[72] Y. Park, C. Depeursinge, and G. Popescu, Nature Photonics 12, 578 (2018).

[73] V. Emery and S. Kivelson, Nature 374, 434 (1995).

[74] W. H. Louisell, Physics Letters 7 (1963).

[75] K. Husimi, Proceedings of the Physico-Mathematical Society of Japan [Nippon Sugaku-Buturigakkwai Kizi Dai 3 Ki] 22, 264 (1940).

[76] K. Thapliyal, S. Banerjee, and A. Pathak, Annals of Physics 366, 148 (2016).

[77] P. Gupta and A. Pathak, Physics Letters A 365, 393 (2007).

[78] R. Demkowicz-Dobrzański, M. Jarzyna, and J. Kołodyński, in Progress in Optics, Vol. 60 (Elsevier, 2015) pp. 345-435.

[79] K. Thapliyal, N. L. Samantray, J. Banerji, and A. Pathak, Physics Letters A 381, 3178 (2017). 
[80] S. Banerjee and R. Srikanth, Modern Physics Letters B 24, 2485 (2010).

[81] R. Srikanth and S. Banerjee, The European Physical Journal D-Atomic, Molecular, Optical and Plasma Physics 53, 217 (2009).

[82] R. Srikanth and S. Banerjee, Physics Letters A 374, 3147 (2010).

[83] Z. Ou, Physical Review A 55, 2598 (1997).

\section{APPENDIX: PHASE ESTIMATION}

The variance of the difference in the photon numbers in the two output modes of the Mach-Zehnder interferometer for input PADFS and PSDFS are

$$
\begin{aligned}
\left(\Delta J_{z}\right)_{+}^{2} & =\cos ^{2} \phi\left\{\frac{1}{2} \frac{\left|N_{+}\right|^{2}}{n !} \sum_{p, p^{\prime}=0}^{n}\left(\begin{array}{l}
n \\
p
\end{array}\right)\left(\begin{array}{c}
n \\
p^{\prime}
\end{array}\right)\left(-\alpha^{\star}\right)^{(n-p)}(-\alpha)^{\left(n-p^{\prime}\right)} \exp \left[-|\alpha|^{2}\right]\right. \\
& \times \sum_{m=0}^{\infty} \frac{\alpha^{m}\left(\alpha^{\star}\right)^{m+p-p^{\prime}}(m+p+u) !}{m !\left(m+p-p^{\prime}\right) !}\left[\frac{1}{2}(m+p+u)(m+p+u-1)+(m+p+u)-\frac{1}{2}\right. \\
& \left.\left.\times \frac{\left|N_{+}\right|^{2}}{n !} \sum_{p, p^{\prime}=0}^{n}\left(\begin{array}{l}
n \\
p
\end{array}\right)\left(\begin{array}{c}
n \\
p^{\prime}
\end{array}\right)\left(-\alpha^{\star}\right)^{(n-p)}(-\alpha)^{\left(n-p^{\prime}\right)} \exp \left[-|\alpha|^{2}\right] \sum_{m=0}^{\infty} \frac{\alpha^{m}\left(\alpha^{\star}\right)^{m+p-p^{\prime}}(m+p+u) !(m+p+u)^{2}}{m !\left(m+p-p^{\prime}\right) !}\right]\right\} \\
& +\sin ^{2} \phi\left\{\frac{1}{4} \frac{\left|N_{+}\right|^{2}}{n !} \sum_{p, p^{\prime}=0}^{n}\left(\begin{array}{l}
n \\
p
\end{array}\right)\left(\begin{array}{c}
n \\
p^{\prime}
\end{array}\right)\left(-\alpha^{\star}\right)^{(n-p)}(-\alpha)^{\left(n-p^{\prime}\right)} \exp \left[-|\alpha|^{2}\right] \sum_{m=0}^{\infty} \frac{\alpha^{m}\left(\alpha^{\star}\right)^{m+p-p^{\prime}}(m+p+u) !(m+p+u)}{m !\left(m+p-p^{\prime}\right) !}\right\},
\end{aligned}
$$

and

$$
\begin{aligned}
& \left(\Delta J_{z}\right)_{-}^{2}=\cos ^{2} \phi\left\{\frac{1}{2} \frac{\left|N_{-}\right|^{2}}{n !} \sum_{p, p^{\prime}=0}^{n}\left(\begin{array}{l}
n \\
p
\end{array}\right)\left(\begin{array}{c}
n \\
p^{\prime}
\end{array}\right)\left(-\alpha^{\star}\right)^{(n-p)}(-\alpha)^{\left(n-p^{\prime}\right)} \exp \left[-|\alpha|^{2}\right]\right. \\
& \times \sum_{m=0}^{\infty} \frac{\alpha^{m}\left(\alpha^{\star}\right)^{m+p-p^{\prime}}(m+p) !(m+p) !}{m !\left(m+p-p^{\prime}\right) !(m+p-v) !}\left[\frac{1}{2}(m+p-v)(m+p-v-1)+(m+p-v)-\frac{1}{2}\right. \\
& \left.\left.\times \frac{\left|N_{-}\right|^{2}}{n !} \sum_{p, p^{\prime}=0}^{n}\left(\begin{array}{c}
n \\
p
\end{array}\right)\left(\begin{array}{c}
n \\
p^{\prime}
\end{array}\right)\left(-\alpha^{\star}\right)^{(n-p)}(-\alpha)^{\left(n-p^{\prime}\right)} \exp \left[-|\alpha|^{2}\right] \sum_{m=0}^{\infty} \frac{\alpha^{m}\left(\alpha^{\star}\right)^{m+p-p^{\prime}}(m+p) !(m+p-v)^{2}}{m !\left(m+p-p^{\prime}\right) !(m+p-v) !}\right]\right\} \\
& +\sin ^{2} \phi\left\{\frac{1}{4} \frac{\left|N_{-}\right|^{2}}{n !} \sum_{p, p^{\prime}=0}^{n}\left(\begin{array}{c}
n \\
p
\end{array}\right)\left(\begin{array}{c}
n \\
p^{\prime}
\end{array}\right)\left(-\alpha^{\star}\right)^{(n-p)}(-\alpha)^{\left(n-p^{\prime}\right)} \exp \left[-|\alpha|^{2}\right] \sum_{m=0}^{\infty} \frac{\alpha^{m}\left(\alpha^{\star}\right)^{m+p-p^{\prime}(m+p) !(m+p) !(m+p-v)}}{m !\left(m+p-p^{\prime}\right) !(m+p-v) !}\right\},
\end{aligned}
$$

respectively. These analytical expressions are obtained using the higher-order moment defined in Eqs. (6) and (7) of Ref. [20]. Similarly, the other parameter required in Eq. (19) for PADFS and PSDFS are obtained as

$$
\left(\frac{d\left\langle\hat{J}_{z}\right\rangle}{d \phi}\right)_{+}=\frac{1}{2} \frac{\left|N_{+}\right|^{2}}{n !} \sum_{p, p^{\prime}=0}^{n}\left(\begin{array}{c}
n \\
p
\end{array}\right)\left(\begin{array}{c}
n \\
p^{\prime}
\end{array}\right)\left(-\alpha^{\star}\right)^{(n-p)}(-\alpha)^{\left(n-p^{\prime}\right)} \exp \left[-|\alpha|^{2}\right] \sum_{m=0}^{\infty} \frac{\alpha^{m}\left(\alpha^{\star}\right)^{m+p-p^{\prime}}(m+p+u) !}{m !\left(m+p-p^{\prime}\right) !}(m+p+u) \sin \phi
$$

and

$$
\left(\frac{d\left\langle\hat{J}_{z}\right\rangle}{d \phi}\right)_{-}=\frac{1}{2} \frac{\left|N_{-}\right|^{2}}{n !} \sum_{p, p^{\prime}=0}^{n}\left(\begin{array}{l}
n \\
p
\end{array}\right)\left(\begin{array}{c}
n \\
p^{\prime}
\end{array}\right)\left(-\alpha^{\star}\right)^{(n-p)}(-\alpha)^{\left(n-p^{\prime}\right)} \exp \left[-|\alpha|^{2}\right] \sum_{m=0}^{\infty} \frac{\alpha^{m}\left(\alpha^{\star}\right)^{m+p-p^{\prime}}(m+p) !(m+p) !}{m !\left(m+p-p^{\prime}\right) !(m+p-v) !}(m+p-v) \sin \phi,
$$

respectively. 